\title{
Direct etching at the nanoscale through nanoparticle-directed capillary condensation.
}

\author{
M. Garín, ${ }^{1,2 *}$ R. Khoury, ${ }^{3}$ I. Martín, ${ }^{1}$ E.V. Johnson ${ }^{3}$ \\ ${ }^{1}$ Grup de recerca en Micro i Nanotecnologies, Departament d'Enginyeria Electrònica, \\ Universitat Politècnica de Catalunya, c/ Jordi Girona Pascual 1-3, Barcelona 08034, Spain \\ 2 GR-MECAMAT, Department of Engineering, Universitat de Vic - Universitat Central de Catalunya, c/ de la \\ Laura 13, 08500 Vic, Spain \\ 3 Laboratoire de Physique des Interfaces et des Couches Minces (LPICM), CNRS. \\ Ecole Polytechnique, Institut Polytechnique de Paris, 91128 Palaiseau, France. \\ * Corresponding author: moises.garin@uvic.cat
}

\begin{abstract}
We report on a method to locally deliver at the nanoscale a chemical etchant in vapor phase by capillary condensation in a meniscus at a nanoparticle/substrate interface. The process is simple, scalable and does not require functionalization of the nanoparticles. Furthermore, it does not rely on any specific chemical properties of the materials other than the solution being aqueous and the wettability properties of the surfaces involved, which should enable its application to other materials and chemicals combinations. In particular, in this work we demonstrate the proposed process by periodically pattering a $\mathrm{SiO}_{2}$ layer using a self-assembled monolayer of polystyrene particles exposed to $\mathrm{HF}$ vapor. The patterned $\mathrm{SiO}_{2}$ layer is then used as a mask to etch a pattern of inverted nanopyramids in $\mathrm{Si}$. The silicon nanopatterning has been demonstrated for particles sizes from $800 \mathrm{~nm}$ down to $100 \mathrm{~nm}$, achieving pyramids with a size down to $50 \mathrm{~nm}$ for $100 \mathrm{~nm}$ nanoparticles.
\end{abstract}

\section{Introduction}

The ability to pattern and structure materials at the nanoscale has enabled a rapid expansion of nanotechnology during the past decades. In particular, not only is it essential for the microelectronic industry ${ }^{1}$ but also for the development of metamaterials, ${ }^{2,3}$ metasurfaces, ${ }^{4}$ plasmonics ${ }^{5}$ and nanophotonics, ${ }^{6}$ just to name a few examples. Although some applications can benefit from random nanostructuring techniques, ${ }^{7}$ sophisticated and expensive nanolithography technologies are typically required, such as extreme UV lithography, ${ }^{8}$ electron beam lithography, ${ }^{9}$ ion beam lithography, ${ }^{10}$ nanoimprint lithography ${ }^{11}$ or scanning probe lithography. ${ }^{12,13}$ In addition to these, alternative lowcost self-assembly nanopatterning methods have been proposed such as block copolymers ${ }^{14}$ and, most relevant to this work, colloidal lithography. ${ }^{15-20}$

Colloidal lithography, sometimes called nanosphere lithography, relies on colloidal solutions of, most typically, polystyrene or silica micro-/nano-spheres with a very low size dispersion. These nanospheres can then be self-assembled over large areas, either flat or irregular, ${ }^{15}$ adopting a closepacked arrangement that will act either as shadow mask for deposition ${ }^{18}$ or as etching mask. ${ }^{19}$ Due to its characteristics, colloidal lithography is usually combined with dry etching techniques.

Reversing the pattern, i.e. etching under the spheres, requires extra steps usually including sphere shrinking and depositing a sacrificial layer. Recently, it has been proposed that functionalized microspheres can be used to locally promote etching around the particles. ${ }^{20}$ In this way, silicon pyramids were etched into silicon at the locations defined by single particles. However, this method 
seems to work at a particle size scale and, therefore, it does not seem feasible to apply it on a closepacked self-assembled layer as it will most probably lead to the etching of the whole surface.

In this paper we demonstrate the use of nanospheres to locally deliver etchant in vapor phase by capillary condensation at the bottom of the spheres. By precisely controlling the partial pressures of water and etchant in vapor phase, the etching can be promoted in a region much smaller than the actual size of the particle and, as a result, the technique can be used with close-packed arrangements of nanospheres. As a demonstration, we have used the process to pattern a layer of $\mathrm{SiO}_{2}$ with an array of nanoholes by using hydrogen fluoride (HF) vapor. This layer is then used as a mask to create different arrays of nanopyramids in silicon with pyramid sizes as small as $50 \mathrm{~nm}$ in size and $100 \mathrm{~nm}$ in pitch.

\section{Results and discussion}

The samples were prepared as follows. First, a 30nm dry oxide was thermally grown on a standard polished silicon $<100>$ oriented wafer, which was then cleaved into small samples of $1 \mathrm{~cm}^{2}$. A close-packed monolayer of polystyrene nanoparticles (PS-NPs) was then deposited over the surface of the samples using the floating-transfer technique. ${ }^{21}$ Four different nominal particle sizes were used namely $800 \mathrm{~nm}, 600 \mathrm{~nm}, 400 \mathrm{~nm}$ and $100 \mathrm{~nm}$, which were obtained as a 5\% aqueous suspension (Microparticles $\mathrm{GmbH}$ ) and that were further diluted in an equal volume of ethanol as a spreading agent. The monolayers were created at the water/air interface inside a petri dish, transferred onto the surface of the substrates, and allowed to dry in ambient air conditions. This process is shown schematically in Figure $1 \mathrm{a}-\mathrm{c}$, while figure $1 \mathrm{~d}$ shows a photograph of two typical monolayer samples.

Once dry, the samples were etched in HF vapor ambient, as follows. The etching was performed in a home-made set-up (see Fig. 1e) at $25^{\circ} \mathrm{C}$ and under atmospheric pressure conditions. Due to the HF toxicity, the set-up was placed in a fume hood to avoid accidental exposure to both HF spills and HF vapors. The reaction chamber has a volume of $1 \mathrm{~L}$, and its ambient is controlled by mixing dry $\mathrm{N}_{2}$ and $\mathrm{N}_{2}$ saturated with $\mathrm{HF}+\mathrm{H}_{2} \mathrm{O}$ vapors at specific flow rates. Saturation is achieved by bubbling $\mathrm{N}_{2}$ through a $5 \% \mathrm{HF}$ aqueous solution at room temperature, which should reach a partial equilibrium pressure of $22.6 \mathrm{mmHg}$ for $\mathrm{H}_{2} \mathrm{O}$ and $0.11 \mathrm{mmHg}$ for $\mathrm{HF}^{22}$ representing a $\mathrm{HF}$ concentration of $147 \mathrm{ppm}$ in the saturated flow. The sample is placed in the reaction chamber and is subjected to four cycles following the following three-step pattern: a) $10 \mathrm{~min}$ with $140 \mathrm{sccm}$ of saturated $\mathrm{N}_{2}$, b) 10 min with $140 \mathrm{sccm}$ of saturated $\mathrm{N}_{2}+200 \mathrm{sccm}$ of dry $\mathrm{N}_{2}$, and c) 10 min with $200 \mathrm{sccm}$ dry $\mathrm{N}_{2}$. During this process, a HF-rich meniscus form between the particles and the sample surface (Fig. 1f), which locally etches the SiO2 layer (Fig. 1g). After the four cycles the PS monolayer is removed from the sample surface and inverted pyramids are created by immersing the sample $5 \mathrm{~min}$. in $25 \%$ tetramethylammonium hydroxide (TMAH) solution (25\%) at $85{ }^{\circ} \mathrm{C}$ (Fig. $1 \mathrm{~h}$ ). Just before the TMAH etch, we performed a short dip (10") in HF (5\%) to remove the native oxide from the opened pattern. Figure 2 shows scanning electron microscope (SEM) images, top view, of both the initial close-packed monolayer of spheres and the arrays of inverted pyramids obtained after the process. Further details and comments on the inverted pyramid formation is included in the supplementary information. 

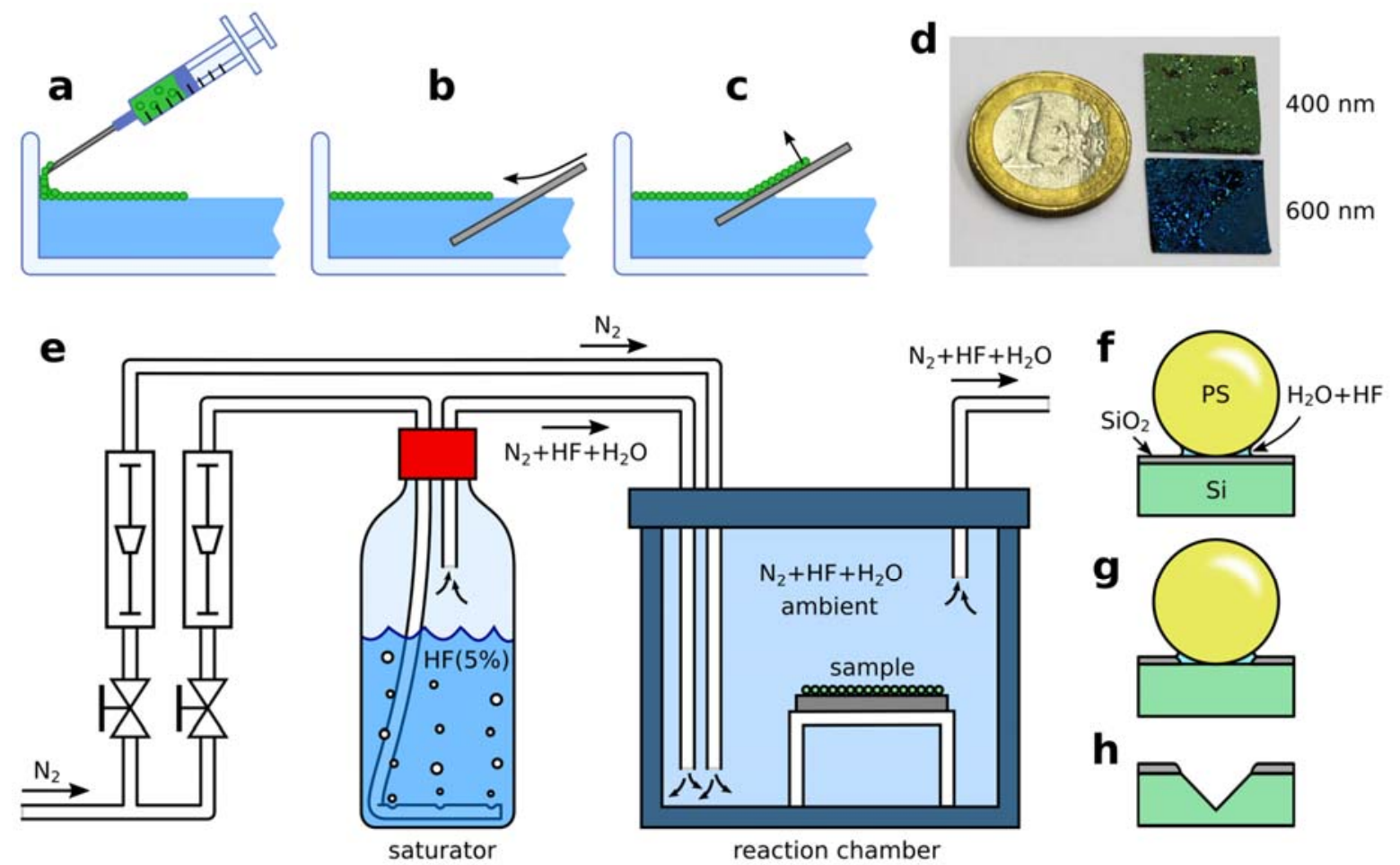

Figure 1. $(\mathrm{a}-\mathrm{c})$ Schematic of close-packed monolayer sample preparation. (d) Photography of typical samples after monolayer preparation. (e) Schematic of the HF-vapor etching set-up. (f-g) Schematic of the nanotexturization process: formation of a HF-rich meniscus (f), local etching of the $\mathrm{SiO}_{2}(\mathrm{~g})$ and formation of pyramids in standard TMAH solution (h).
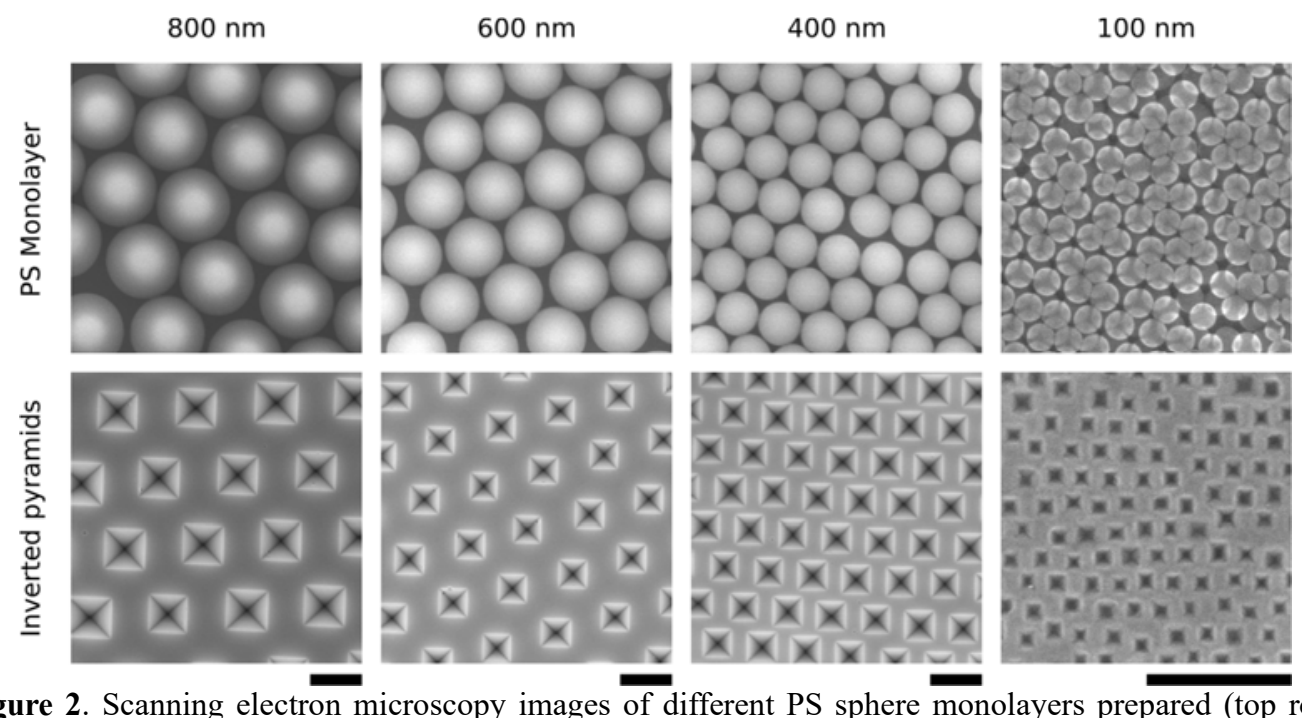

Figure 2. Scanning electron microscopy images of different PS sphere monolayers prepared (top row) and resulting pyramid arrays after full process (bottom row). From left to right, the PS microspheres used had a diameter of $800 \mathrm{~nm}, 600 \mathrm{~nm}, 400 \mathrm{~nm}$ and $100 \mathrm{~nm}$. All scalebars represent 500nm. 
The experimental routine was determined after several tests based on the dynamics of capillary condensation, which is slow and can take several minutes to reach the equilibrium condition. ${ }^{23}$ Additionally, the process is further slowed down by the dynamics of the chamber ambient stabilization after changes in the flow rates. During the first step of each cycle the samples are exposed to a $100 \%$ relative humidity (RH) ambient with $147 \mathrm{ppm}$ of HF, which induces a quick formation of water $+\mathrm{HF}$ menisci at the bottom of the particles, promoting the $\mathrm{SiO}_{2}$ etching there. If this ambient is maintained for a long time, the menisci slowly grow in excess inducing the complete wetting and etching of the surface. Furthermore, even in the absence of nanospheres, HF vapors eventually condensate and etch the $\mathrm{SiO}_{2}$ layer everywhere, ${ }^{24}$ as has been studied in the same setup. To avoid this, the ambient is diluted with dry $\mathrm{N}_{2}$ in the second step, reducing the $\mathrm{RH}$ to $40 \%$ and $\mathrm{HF}$ concentration to $60 \mathrm{ppm}$, approximately. The HF-rich water menisci are maintained in this stage thanks to the hysteresis of the meniscus formation/destruction process. ${ }^{25}$ The last step, only with dry $\mathrm{N}_{2}$ ambient, dries the structure before the next cycle, to avoid accumulation of condensation over several cycles. Although not strictly necessary for the process, we have noticed that the drying step offers a better control of the menisci formation, therefore improving the definition of the etching and slightly reducing the final pyramid sizes.
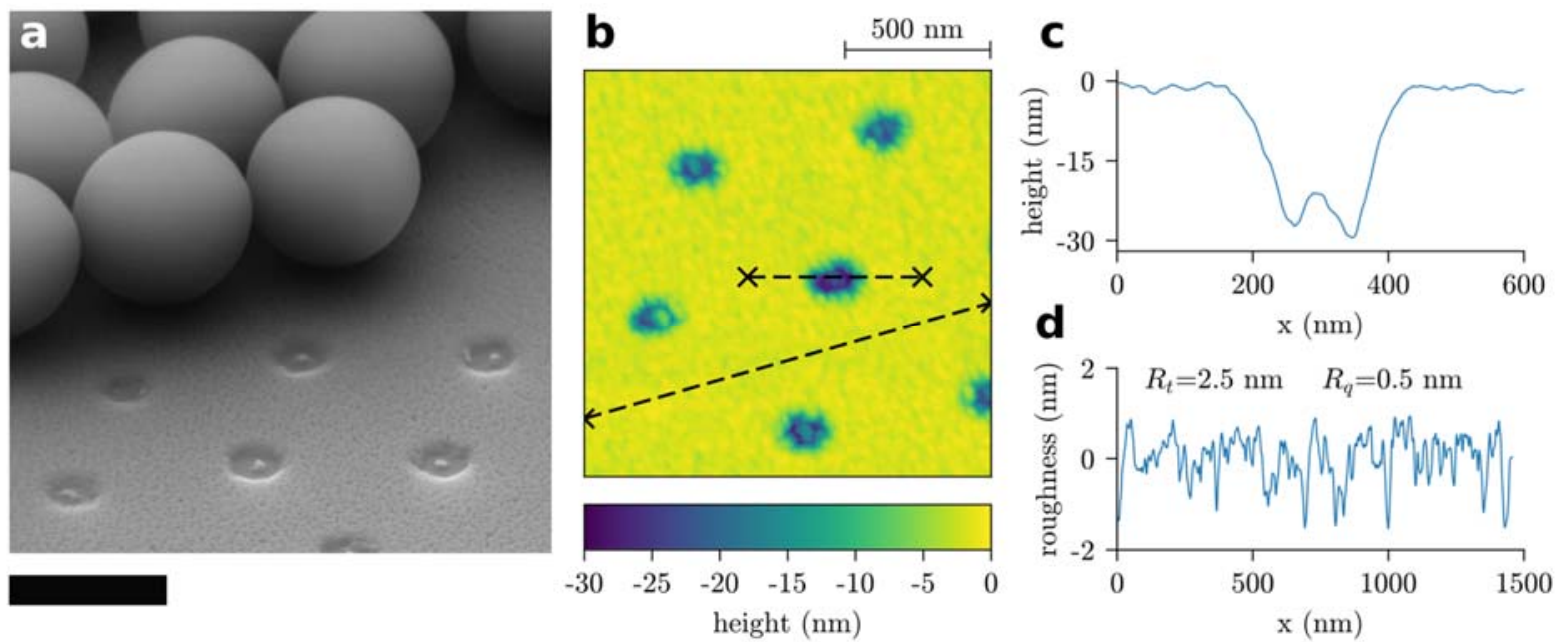

Figure 3. SEM image (a) and AFM image (b) of the $\mathrm{SiO}_{2}$ surface right after $\mathrm{HF}$ vapor etching for a sample with $600 \mathrm{~nm}$ spheres. Panels (c) and (d) shows the surface profiles following paths indicated in the AFM image.

Figure 3a shows an SEM image of the surface of one sample with $600 \mathrm{~nm}$ nanospheres right after the vapor etching (after four cycles of the process), where a few spheres were removed in order to reveal the $\mathrm{SiO}_{2}$ surface. In the SEM image, one notes that at most of the sphere locations, the $30 \mathrm{~nm} \mathrm{SiO} 2$ layer has been completely removed and the $\mathrm{Si}$ surface is clearly visible through the resulting opening. A small $\mathrm{SiO}_{2}$ spot, around $35 \mathrm{~nm}$ in diameter, is still usually present in the center of the etched areas, which we believe is due to a masking effect at the particle/ $\mathrm{SiO}_{2}$ contact point. 
We further investigated the surface of the etched samples through atomic force microscopy (AFM). Figure $3 \mathrm{~b}$ shows an AFM image of a $1.4 \mu \mathrm{m} \times 1.4 \mu \mathrm{m}$ region without nanospheres. The profile through one of the openings is shown in Fig. 3c, revealing a maximum etch depth of $30 \mathrm{~nm}$, also suggesting a complete removal of the oxide layer after four cycles of vapor etching. Notice that the AFM image at the openings is limited by the shape of the tip, which does not allow one to clearly see the bottom of the openings. Furthermore, the height of the $\mathrm{small}^{\mathrm{SiO}_{2}}$ spot is significantly lower than the initial $\mathrm{SiO}_{2}$ layer height. This small spot is not significant for the subsequent process, as any remaining oxide in the etched openings will be removed during the 10 seconds HF dip prior to the TMAH etching, which we estimated to remove around $8 \mathrm{~nm}$ of $\mathrm{SiO}_{2}$. This etching rate estimation was consistently obtained both through ellipsometry measurements and through repeated dipping until completely removing the $\mathrm{SiO}_{2}$ on reference samples.

Another interesting feature that both the SEM and AFM images reveal is the roughening of the remaining $\mathrm{SiO}_{2}$ layer. This roughening can be attributed to the direct etching of the $\mathrm{SiO}_{2}$ by the HF vapors. A roughness analysis of the AFM images, see Fig. 3d, shows a root mean squared (RMS) roughness of $R_{\mathrm{q}}=0.5 \mathrm{~nm}$ and a total roughness $R_{\mathrm{t}}=2.5 \mathrm{~nm}$. This roughening occurred just at the top surface and did not induce any appreciable reduction of the total layer thickness, as the $30 \mathrm{~nm}$ height can still be observed at the openings (Fig. 3c). Furthermore, no thickness difference could be measured through ellipsometry, before and after the HF vapor etching, using a reference sample with $100 \mathrm{~nm} \mathrm{SiO}_{2}$.

The difference in the etching rates between the capillary condensates and the rest of the surface is remarkable, particularly considering that $\mathrm{SiO}_{2}$ is a hydrophilic surface that is easily etched by HF vapors. This etching selectivity induced by the capillary condensation strongly suggests that this technique might be applicable to a broad range of substrates and chemical agents. Finally, it is worth pointing out that the progressive roughening of the $\mathrm{SiO}_{2}$ surface is a critical aspect. It enhances condensation and, therefore, increases the etching rate of $\mathrm{SiO}_{2}$, which might eventually become a limiting factor to the selectivity of the process. This effect distinctly manifests when exposing oxidized samples just to $\mathrm{N}_{2}$ ambient with $\mathrm{RH}>90 \%$ and $\mathrm{HF}$ vapors (step 1 conditions, without any drying step) but for an extended time. Under these conditions the sample eventually develops a thick liquid layer visible to the naked eye that eventually etches the entire $\mathrm{SiO}_{2}$ layer. In contrast, no such liquid layer is observable after exposing the sample to a similar ambient but without HF. We strongly believe that this difference manifests after an initial surface roughening by HF vapor, which then speeds up condensation and droplet formation. 

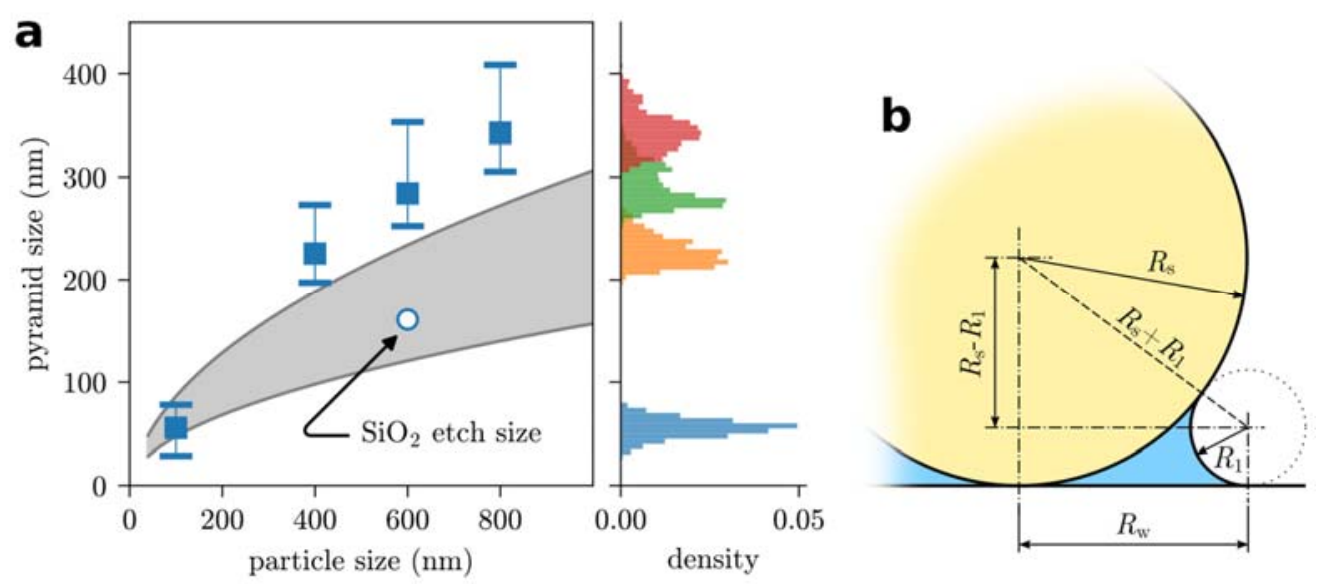

Figure 4. (a) Pyramid size distribution obtained. Squares represent the median of the distribution. As a reference, the size of the $\mathrm{SiO}_{2}$ opening after etching for $600 \mathrm{~nm}$ particles is shown as a circle. The gray shaded area represents the calculated size of the meniscus for RH in the range $80 \%$ (bottom) to $95 \%$ (top). (b) Schematic of the meniscus geometry considered for calculations.

We have characterized the size of the pyramids obtained with the different colloids through SEM imaging. Pyramid sizing was automatized for the samples with $400 \mathrm{~nm}, 600 \mathrm{~nm}$ and $800 \mathrm{~nm}$ particles; however, it needed to be performed manually for the $100 \mathrm{~nm}$ sample. Roughly, the number of pyramids measured ranged from 400 for the manual sizing to 1000 when using the automatic image analysis. Results are shown in Figure 4, where the squares mark the mode of the size distribution, while bars indicate the full range of the data. The measured size distribution is also shown on the right for convenience. As the results clearly show, there is a direct dependence between the particle and the final pyramid sizes; the larger the particle the larger the final pyramid. More precisely, the size of the pyramids depends on the size of the HF-rich water meniscus that forms between the particle and the sample, which, in turn, depends on the size of the particle as well as the ambient relative humidity and contact angles at the $\mathrm{SiO}_{2}$ and PS surfaces. Figure $4 \mathrm{~b}$ shows schematically the geometry of the condensed water meniscus, where the nodoid shape of the meniscus is simplified by a circular shape with radius $R_{1}$, as it is common practice. For the sake of simplicity, since we are in a non-equilibrium situation at the nanoscale, we will further assume that the contact angle is zero on both the substrate and the particle. The radius of the wetted $\mathrm{SiO}_{2}$ region can, then, be calculated as

$$
R_{w}=\sqrt{\left(R_{s}+R_{1}\right)^{2}-\left(R_{s}-R_{1}\right)^{2}}=2 \sqrt{\frac{R_{s}}{k}}
$$

where $R_{\mathrm{S}}$ is the radius of the nanoparticle and $k=1 / R_{1}$ is the curvature of the meniscus. The curvature of the meniscus can be related to the RH through the Laplace-Kelvin equation:

$$
\gamma k=\frac{R T}{V_{\mathrm{m}}} \ln \left(\frac{p}{p_{0}}\right)
$$

where $\gamma$ is the surface tension, $R$ is the universal gas constant, $T$ is the temperature, $V_{\mathrm{m}}$ is the molar volume of the liquid phase, $p$ is the vapor pressure and $p_{0}$ is the saturation vapor pressure. Assuming that the HF concentration is low enough so that the typical water constants can be used, with the 
relative humidity $\mathrm{RH}=p / p_{0}$, equations 1 and 2 readily allow one to calculate the size of the etched $\mathrm{SiO}_{2}$ region as a function of particle size and $\mathrm{RH}$. In figure $4 \mathrm{a}$ we have included in gray the diameter of the wetted region for a RH in the range $80 \%$ to $96 \%$. The experimental size of the pyramids vs. the particle size follow a similar trend to the size of the meniscus, but with larger sizes. Notice that the whole distribution of pyramid sizes is expected to be above the wetting size as it can be easily by the TMAH etching process, i.e. by the irregularities of the openings and the slight underetching, as well as due to the effect of the 10" HF dip done right before the pyramid formation. In figure $4 \mathrm{a}$ we have also included (open circles) the size of the $\mathrm{SiO}_{2}$ etched area measured right after the vapor etching for the $600 \mathrm{~nm}$ sample (see fig. 3 ). This size, smaller than the final pyramid sizes, is perfectly consistent with the theoretical estimations and coincides, roughly, with the meniscus obtained for a $\mathrm{RH}=90 \%$. Notice that, although beyond the scope of this work, figure 4a strongly suggest that the size of the pyramids can probably be controlled through a fine control of the RH during the vapor-phase treatment.

Finally, it should be mentioned that the typical pyramid size for the $100 \mathrm{~nm}$ case is smaller than expected. Although this deviation could just flag the limits of the simple model considered, it also could be attributed to the fact that the process only worked in a small portion of the sample surface in that particular case, while the process worked flawlessly for the rest of particle sizes. Therefore, the average pyramid size for $100 \mathrm{~nm}$ particles is not representative of the standard process conditions. The difficulties we experienced with the $100 \mathrm{~nm}$ particles could most probably be related to the lower quality monolayers that we were able to produce (see fig. 2): particles were not perfectly close packed and a double layer was mostly obtained. On the other hand, it may also indicate that the process conditions for the HF-vapor treatment need a slight fine tuning for the smallest particles size.

\section{Conclusions}

In this work we have introduced a method to locally deliver, with nanoscale resolution, a chemical in vapor phase through capillary condensation. In particular, the chemicals condensate in the meniscus at a nanoparticle/substrate interface. The process is simple, scalable, does not require functionalizing the nanoparticles, and it might potentially be applicable to different materials and chemicals. As a proof of concept, in this work we demonstrate the proposed process by periodically pattering a thin $\mathrm{SiO}_{2}$ layer using a self-assembled monolayer of polystyrene particles exposed to $\mathrm{HF}$ vapor. The patterned $\mathrm{SiO}_{2}$ layer is then used as a mask to create an arrangement of inverted nanopyramids on $\mathrm{Si}$. The silicon nanopatterning has been demonstrated for particle sizes from $800 \mathrm{~nm}$ down to $100 \mathrm{~nm}$ using the exact same process conditions for all samples, demonstrating the robustness and repeatability of the process.

\section{Acknowledgments}

This work has been financially supported through the following projects: ENE2015-74009JIN funded by the Spanish Ministry of Economy and Competitiveness (MINECO) and the European Regional Development Fund (ERDF-EU), TEC2017-82305-R (MINECO), the InnoEnergy PhD School Programme and the European Institute of Technology (EIT). 


\section{References}

1 H. J.M. Veendrick, Nanometer CMOS ICs, From basics to ASICs, $2^{\text {nd }}$ edition, Springer, 2017.

2 Y. Liu and X. Zhang, "Metamaterials: a new frontier of science and technology" Chem. Soc. Rev., 2011, 40, 2494-2507.

3 S. Jahani and Z. Jacob "All-dielectric metamaterials" Nat. Nanotech., 2016, 11, 23-36.

4 M. Khorasaninejad and F. Capasso "Metalenses: Versatile multifunctional photonic component" Science, 2017, 358, 1146.

5 W. L. Barnes, A. Dereux, and T. W. Ebbesen "Surface plasmon subwavelength optics" Nature 424, 824-830 (2003).

6 Y. Shen, C. S. Friend, Y. Jiang, D. Jakubczyk, J. Swiatkiewicz and P. N. Prasad "Nanophotonics: Interactions, Materials, and Applications" J. Phys. Chem. B, 2000, 104, 7577 7587.

7 X. Liu, P. R. Coxon, M. Peters, B. Hoex, J. M. Cole and D. J. Fray, "Black silicon: fabrication methods, properties and solar energy applications" Energy Environ. Sci., 2014, 7, 3223.

8 C. Wagner, and N. Harned "EUV lithography: Lithography gets extreme" Nat. Photonics, 2010, 4, 24-26.

9 V. R. Manfrinato, L. Zhang, D. Su, H. Duan, R. G. Hobbs, E. A. Stach and K. K. Beergg "Resolution Limits of Electron-Beam Lithography toward the Atomic Scale" Nano Lett., 2013, 13, 1555-1558.

10 Z. Liu, A. Shah, T. Alasaarela, N. Chekurov, H. Savin and I. Tittonen "Silicon dioxide mask by plasma enhanced atomic layer deposition in focused ion beam lithography" Nanotechnology, 2017, 28, 085303.

11 S. Y. Chou, P. R. Krauss and P. J. Renstrom "Imprint Lithography with 25-Nanometer Resolution” Science 272, 85-87 (1996).

12 R. D. Piner, J. Zhu, F. Xu, S. Hong and C. A. Mirkin “«Dip-Pen» Nanolithography” Science, 1999, 283, 661-663.

13 R. Garcia, A. W. Knoll and E. Riedo "Advanced scanning probe lithography" Nat. Nanotechnol., 2014, 9, 577-587.

14 C. M. Bates, M. J. Maher, D. W. Janes, C. J. Ellison and C. G. Willson, "Block Copolymer Lithography" Macromolecules, 2014, 47, 2-12.

15 S. P. Bhawalkar, J. Qian, M. C. Heiber and L. Jia "Development of a Colloidal Lithography Method for Patterning Nonplanar Surfaces” Langmuir, 2010, 26, 16662-16666.

16 O. D. Velev and S. Gupta "Materials Fabricated by Micro- and Nanoparticle Assembly - The Challenging Path from Science to Engineering” Adv. Mater., 2009, 21, 1897-1905.

17 P. Colson, C. Henrist and R. Cloots "Nanosphere Lithography: A Powerful Method for the Controlled Manufacturing of Nanomaterials" J. Nanomater., 2013, 2013, 948510.

18 K. Chen, T. D. Dao and T. Nagao "Tunable Nanoantennas for Surface Enhanced Infrared Absorption Spectroscopy by Colloidal Lithography and Post-Fabrication Etching" Sci. Rep., 2017, 7, 44069.

19 C. L. Cheung, R. J. Nikolić, C. E. Reinhardt and t. F. Wang "Fabrication of nanopillars by nanosphere lithography" Nanotechnology, 2006, 17, 1339-1343. 
20 N. Chaturvedi, E. Hsiao, D. Velegol and S. H. Kim "Maskless Fabrication of Nanowells Using Chemically Reactive Colloids" Nano Lett., 2011, 11, 672-676.

21 J. R. Oh, J. H. Moon, S. Yoon, C. R. Park and Y. R. Do "Fabrication of wafer-scale polystyrene photonic crystal multilayers via the layer-by-layer scooping transfer technique" J. Mater. Chem., 2011, 21, 14167.

22 J. C. Brosheer, F. A. Lenfesty and K. L. Elmore "Vapor pressure of hydrofluoric acid solutions" Ind. Eng. Chem., 1947, 39, 423-427.

23 B. L. Weeks and J. J. DeYoreo "Dynamic meniscus growth at a scanning probe tip in contact with a gold substrate" J. Phys. Chem. B, 2006, 110, 10231-10233.

24 C. R. Helms and B. E. Deal "Mechanics of the $\mathrm{HF} / \mathrm{H}_{2} \mathrm{O}$ vapor phase etching of $\mathrm{SiO}_{2}$ " J. Vac. Sci. Technol. A, 1991, 10, 806-811.

25 B. L. Weeks and M. W. Vaughn "Direct imaging of meniscus formation in atomic force microscopy using environmental scanning electron microscopy" Langmuir, 2005, 21, 80968098. 